\title{
NONEQUILIBRIUM EFFECTS IN A BIMOLECULAR CHEMICAL REACTION IN A DILUTE GAS
}

\author{
A.S. CUKrowski ${ }^{a}$, S. Fritzsche ${ }^{b}$, AND J. PoplelaWsik ${ }^{a}$ \\ ${ }^{a}$ Institute of Physical Chemistry, Polish Academy of Sciences \\ Kasprzaka 44/52, 01-224 Warszawa, Poland \\ ${ }^{b}$ Research Group Statistical Theory of Non-Equilibrium Processes \\ WIP, KAI e.V., Permoser Str. 15, O-7050 Leipzig, Germany
}

(Received April 15, 1993)

\begin{abstract}
Perturbation solution of the Boltzmann equation for a dilute gas with a chemical reaction $A+A \rightarrow B+B$ is presented. Analytical results for the nonequilibrium effects on the rate of chemical reaction are obtained for the line-of-centers model. It is shown that taking into account the energy transfer from reagents $A$ to products $B$ permits to get new results. The nonequilibrium corrections obtained from these results are much larger than those obtained with neglecting tlis energy transfer. These results are verified by a comparison with the numerical results obtained from the modified Nanbu-Babovsky Monte Carlo computer simulations.
\end{abstract}

PACS numbers: 82.20.-w, 82.20.Mj, 82.20.Wt, 82.30.Eh, 05.20.Dd

\section{Introduction}

In a dilute gas in which a bimolecular reaction takcs place the Maxwell-Boltzmann velocity distribution function changes to nonequilibrium distribution. Prigogine and co-workers [1, 2] have used the perturbation Chapman-Enskog $[3,4]$ method of solution of the Boltzmann equation and they have shown that this effect diminishes the rate constant of chemical reaction. Present $[5,6]$ has analyzed this effect for the reactive cross-section described by the line-of-centers model, which is very suitable for such an analysis. In analytical results for the rate constant of chemical reaction, described by this model with total neglecting of products, the nonequilibrium effects do not exceed $8 \%$, whereas for the Maxwell-Boltzmann distribution function this model leads to the Arrhenius expression [5, 6]. Shizgal and Karplus [7] have confirmed the results of Present. Fitzpatrick and Desloge [8] have shown that neglecting of products has a small effect on such results.

However, as it can be seen from the review written by Popielawski [9], for chemical reactions the results obtained from the perturbation method of solution of 
the Boltzmann equation, contrary to expressions for viscosity, thermal conductivity and diffusion coefficients, cannot be directly compared with experimental data. That is why, recently other possibilities of verification of the results obtained by the perturbation method in this case have been used. Namely, for chemical reaction proceeding in the Lorentz gas, reasonable comparison between such results and the results obtained from the exact numerical solution of the Fokker-Planck equation [10-12] has been performed. We also decided to compare the results obtained from the perturbation solution for the bimolecular chemical reaction in the dilute gas with the results from the Monte Carlo computer simulations of solution of the Boltzmann equation for this reaction. We have cliosen the modified Nanbu-Babovsky direct simulation method [13-19] because this method has been very effective in comparison between analytical and numerical results in the analysis of relaxation of translational energy in a dilute gas [20-22]. Introducing an additional term in the perturbation solution of the Boltzmann equation we have shown [23] that the nonequilibrium effect on the rate constant of chemical reaction can be much larger than in Refs. [5-8] and that similarly as in the Lorentz gas [10-11] the results are accurate for slow reactions only. These results [23] have been also confirmed by the molecular dynamics simulation method developed by Górecki [24-26].

As our results [23] looked interesting, we decided to analyze them more carefully before a final publication. For this purpose we concentrated our attention on the more careful analysis of our treatment of the perturbation method and on improvement of the accuracy of numerical results. The first part of these results connected with perturbation of thermal equilibrium by a simple chemical reaction has been already presented [27]. Recently, the results from Ref. [23] have been confirmed by a simple theoretical analysis [28] assuming that nonequilibrium effects are mainly resulting from a change of temperature in the Maxwell-Boltzmann velocity distribution function. For slow chemical reactions our analytical results from Ref. [23] have been also confirmed by Górecki [29] who used the so-called phenomenological kinetic equations and verified them by molecular dynamics simulations.

In this paper we present the theoretical treatment in the form devcloped from the version which was improved by Prof. Popiclawski before his sudden death and the results of computer simulations performed with a better accuracy than in Ref. [23].

Our paper is organized as follows: In Sec. 2 we formulate the Boltzmann equation for the chemical reaction described. In Sec. 3 we describe the perturbation method of solution of the Boltzmann equation. In Sec. 4 we present for the line-of-centers model the analytical results for the change of the rate constant of chemical reaction due to nonequilibrium effects, whereas in Sec. 5 we show for a comparison such results obtained in traditional way. After a description of the system used for the modified Nanbu-Babovsky Monte Carlo computer simulations in Sec. 6, in Sec. 7 we compare our new analytical results with those obtained in the traditional way, i.e. with the total neglecting of the products of reaction as well as with the numerical results from Sec 6. Finally, in Sec. 8 we discuss the results obtained and make conclusions. 


\section{The Boltzmann equation for a gas with chemical reaction}

We consider the following bimolecular reaction in the dilute gas:

$$
A+A \rightarrow B+B
$$

and neglect all heat effects of this reaction, i.e. we assume that this reaction is neither exothermal nor endothermal one. Ilowever, we will take into consideration that, even in the simplest models of reactive collisions, the particles of product $B$ need not have the same average kinetic energy as the particles of reagents $A$. Assuming that the system is well stirred we can write down the following Boltzmann equation [1]:

$$
\begin{aligned}
& \frac{\partial f_{A}}{\partial t}=\iint\left(f_{A}^{\prime} f_{A_{1}}^{\prime}-f_{A} f_{A_{1}}\right) \sigma_{A A_{1}} g_{A A_{1}} \mathrm{~d} \Omega_{A A_{1}} \mathrm{~d} c_{A_{1}} \\
& -\iint f_{A} f_{A_{1}} \sigma_{A A_{1}}^{*} g_{A A_{1}} \mathrm{~d} \Omega_{A A_{1}} \mathrm{~d} c_{A_{1}}+\iint\left(\int_{A}^{\prime} f_{B}^{\prime}-f_{A} f_{B}\right) \sigma_{A B} g_{A B} \mathrm{~d} \Omega_{A B} \mathrm{~d} c_{B},
\end{aligned}
$$

where $f_{i}$ and $f_{i}^{\prime}$ are the velocity distribution functions for $i$-th component ( $i=$ $A, B$ ). before and after collisions, respectively, $A_{1}$ is introduced to distinguish two molecules of the component $A, t$ is the time, $\sigma_{i j}$ and $\sigma_{i j}^{*}$ are the differential elastic and reactive cross-sections for colliding molecules $i$ and $j, c_{i}$ and $c_{j}$ are their velocities, $g_{i j}=c_{i}-c_{j}, \Omega_{i j}$ denotes the solid angles.

Adapting the usual normalization

$$
\int f_{i} \mathrm{~d} c_{i}=n_{i}, \quad(i=A, B),
$$

where $n_{i}$ is the number density of $i$-th component we introduce the temperature of the system by

$$
T=\frac{2}{3 n k_{\mathrm{B}}}\left[\int f_{A} \frac{1}{2} m_{A} c_{A}^{2} \mathrm{~d} c_{A}+\int f_{B} \frac{1}{2} m_{B} c_{B}^{2} \mathrm{~d} c_{B}\right],
$$

where $n=n_{A}+n_{B}, k_{\mathrm{B}}$ is the Boltzmann constant, $m_{i}$ is the mass of the molecule of $i$-th component. Let us introduce as Shizgal and Karplus [30] the temperatures of components $A$ and $B$

$$
T_{i}=\frac{2}{3 n_{i} k_{\mathrm{B}}} \int f_{i} \frac{1}{2} m_{i} c_{i}^{2} \mathrm{~d} c_{i}, \quad(i=A, B) .
$$

Thus we have

$$
T=\frac{n_{A}}{n} T_{A}+\frac{n_{B}}{n} T_{B}
$$

3. The perturbation solution of the Boltzmann equation for early stages of slow chemical reaction

For early stages of chemical reaction we can assume tlat

$$
n_{A} \gg n_{B}
$$

and write 


$$
\begin{aligned}
\frac{\partial f_{A}}{\partial t}=\int & \int\left(f_{A}^{\prime} f_{A}^{\prime}-f_{A} f_{A_{1}}\right) \sigma_{A A_{1}} g_{A A_{1}} \mathrm{~d} \Omega_{A A_{1}} \mathrm{~d} c_{A_{1}} \\
& -\iint f_{A} \int_{A_{1}} \sigma_{A A_{1}}^{*} g_{A A_{1}} \mathrm{~d} \Omega_{A A_{1}} \mathrm{~d} c_{A_{1}}=-I_{\mathrm{el}}-I_{\text {ree }}
\end{aligned}
$$

where $I_{\mathrm{el}}$ and $I_{\mathrm{re}}$ denote the integrals connected with elastic and reactive collisions, respectively. Equation (8) can be solved by the perturbation method suggested by Prigogine and co-workers [1]. In this method the term due to chemical reaction $I_{\mathrm{re}}$ is treated as a small perturbation. If we assume

$$
f_{A}=f_{A}^{(0)}(T)\left(1+\psi_{A}\right)=f_{A}^{(0)}+f_{A}^{(1)},
$$

where $f_{A}^{(0)}(T)$ is the Maxwell-Boltzmann distribution function with the total temperature $T$ for the system (see Eq. (6))

$$
f_{A}^{(0)}(T)=n_{A}\left(\frac{m_{A}}{2 \pi k_{\mathrm{B}} T}\right)^{3 / 2} \exp \left(-\frac{m_{A} c_{A}^{2}}{2 k_{\mathrm{B}} T}\right)
$$

whereas $\psi_{A}$ and $f_{A}^{(1)}$ are connected with nonequilibrium effects.

We can write Eq. (8) as

$$
D+J=0,
$$

where

$$
\begin{aligned}
& D=\frac{\partial f_{A}}{\partial t}+I_{\mathrm{re}}, \\
& J=I_{\mathrm{el}} .
\end{aligned}
$$

According to the perturbation method we introduce

$$
D^{(0)}=-J^{(1)}
$$

where

$$
\begin{aligned}
D^{(0)}= & \frac{\partial f_{A}^{(0)}}{\partial t}+I_{\mathrm{re}}^{(0)} \\
I_{\mathrm{re}}^{(0)}= & \iint f_{A}^{(0)} f_{A_{1}}^{(0)} \sigma_{A A_{1}}^{*} g_{A A_{1}} \mathrm{~d} \Omega_{A A_{1}} \mathrm{~d} c_{A_{1}}, \\
J^{(1)}=I_{\mathrm{el}}^{(1)}= & \iint\left(f_{A}^{(1)} f_{A_{1}}^{(0)}-f_{A}^{\prime(1)} f_{A_{1}}^{\prime(0)}\right) \sigma_{A A_{1}} g_{A A_{1}} \mathrm{~d} \Omega_{A A_{1}} \mathrm{~d} c_{A_{1}} \\
& +\iint\left(f_{A}^{(0)} f_{A_{1}}^{(1)}-f_{A}^{(0)} f_{A_{1}}^{(1)}\right) \sigma_{A A_{1}} g_{A A_{1}} \mathrm{~d} \Omega_{A A_{1}} \mathrm{~d} c_{A_{1}},
\end{aligned}
$$

where $f_{A}^{(0)}$ and $f_{A}^{(1)}$ are defined by Eqs. (9) and (10). Let us observe that while writing Eqs. (8) and (9) we have not neglected the second term in Eq. (6). This means that in the isolated system it is possible to have the situation in which

$$
T_{B}>T>T_{A} \text {. }
$$

Taking into account the form of Eq. (8) we can write

$$
\frac{\partial f_{A}^{(0)}}{\partial t}=\frac{\partial f_{A}^{(0)}}{\partial n_{A}} \frac{\mathrm{d} n_{A}}{\mathrm{~d} t}+\frac{\partial \int_{A}^{(0)}}{\partial T} \frac{\mathrm{d} T}{\mathrm{~d} \ell},
$$


where

$$
\frac{\mathrm{d} n_{A}}{\mathrm{~d} t}=-\int I_{\mathrm{re}}^{(0)} \mathrm{d} c_{A}
$$

Assuming that the system considered is thermally isolated we can put

$$
\frac{\mathrm{d} T}{\mathrm{~d} t}=0 \text {. }
$$

From Eq. (21) it does not follow that the temperatures of components $A$ and $B$ defined by Eq. (5) have to be constant. As $n$ is constant Eq. (21) leads to the conclusion that

$$
n T=n_{A} T_{A}+n_{B} T_{B}=\text { const. }
$$

Equation (14) is solved by using the expansion of $\psi_{A}$ in the Sonine polynomials

$$
\psi_{A}=\sum_{i} a_{A}^{(i)} S_{1 / 2}^{(i)}\left(\mathcal{C}_{A}^{2}\right)
$$

where

$$
\mathcal{C}_{A}^{2}=\frac{m_{A} c_{A}^{2}}{2 k_{\mathrm{B}} T}
$$

from the condition

$$
\int_{A} f_{A} \mathrm{~d} c_{A}=\int f_{A}^{(0)} \mathrm{d} c_{A}=n_{A}
$$

it follows that

$$
a_{A}^{(0)}=0 \text {. }
$$

As shown by Shizgal and Karplus [30], after taking into account Eqs. (5), (9), (23) and formal properties of the Sonine polynomials, it can be written

$$
T_{A}^{\mathrm{SK}}=T\left(1-a_{A}^{(1)}\right) \text {. }
$$

We have introduced the indices SK to denote by $T_{A}^{S K}$ the Shizgal-Karplus temperature.

Following our derivation from Eqs. (15), and (19)-(21) we can obtain

$$
D^{(0)}=-\frac{f^{(0)}}{n} \int I_{\mathrm{re}}^{(0)} \mathrm{d} c_{\Lambda}+I_{r \mathrm{e}}^{(0)}=-I_{\mathrm{el}}^{(1)} \text {. }
$$

Let us observe that the cocficients $a_{A}^{(i)}$ (see Eqs. (9) and (23)) appear in the right hand side of Eq. (28) only (sce Eqs. (17), (9), and (23)). Therefore, after introducing into the right hand side of Eq. (28) the expansion of $f_{A}$ in the Sonine polynomials (see Eqs. (17), (9) and (23)), multiplying this equation by the appropriate Sonine polynomial and integrating with respect to $\mathrm{d} c_{A}$ we get a linear algebraic equation (if one Sonine polynomial is used) or a set of linear algebraic equations (if more Sonine polynomials are used). After introduction of an appropriate reactive cross-section we can solve such algebraic equations and obtain the results for $a_{A}^{(i)}$, i.e. to have the expressions for nonequilibrium velocity distribution functions (see Eqs. (9), (10) and (23)) from which all necessary macroscopic quantities can be calculated. In the next section we present the results obtained for the line-of-centers model. 


\section{Analytical results for the line-of-centers model}

The rate of chemical rcaction (1) can be obtained by integration of Eq. (8) with respect to $c_{A}$. Taking into account the normalization and conservation of the number of particles in elastic collisions we obtain

$$
v_{A}=-\frac{\mathrm{d} n_{A}}{\mathrm{~d} l}=k_{A} n_{A}^{2},
$$

where

$$
k_{A}=\frac{1}{n_{A}^{2}} \iint f_{A} f_{A_{1}} \sigma_{A A_{1}}^{*} g_{A A_{1}} \mathrm{~d} \Omega_{A A_{1}} \mathrm{~d} c_{A_{1}} .
$$

Equation (30) relates the phenomenological rate constant $k_{A}$ to the microscopic reaction mechanism determined by $\sigma_{A A_{1}}^{*}$. We will analyze the differences between the nonequilibrium values of $k_{A}$ and the equilibrium values of $k_{A}^{(0)}$. These rate constants can be calculated as

$$
k_{A}^{(0)}=\frac{1}{n_{A}^{2}} \iint f_{A}^{(0)} \int_{A_{1}}^{(0)} \sigma_{A A_{1}}^{*} g_{A A_{1}} \mathrm{~d} \Omega_{A A_{1}} \mathrm{~d} c_{A_{1}}
$$

and

$$
\begin{aligned}
k_{A}=\frac{1}{n_{A}^{2}} & \iint f_{A}^{(0)}\left[1+\sum_{i} a_{A}^{(i)} S_{1 / 2}^{(i)}\left(\mathcal{C}_{A}^{2}\right)\right] \\
& \times f_{A_{1}}^{(0)}\left[1+\sum_{i} a_{A_{1}}^{(i)} S_{1 / 2}^{(i)}\left(\mathcal{C}_{A_{1}}^{2}\right)\right] \sigma_{A A_{1}}^{*} g_{A A_{1}} \mathrm{~d} \Omega_{A A_{1}} \mathrm{~d} c_{A} .
\end{aligned}
$$

We adapt the line-of-centers model due to Present [5]

$$
\sigma_{A A_{1}}^{*}= \begin{cases}0, & k \cdot g_{A A_{1}} \leq g^{*}, \\ \frac{1}{4} d_{A}^{2} s_{\mathrm{F}}, & k \cdot g_{A A_{1}}>g^{*},\end{cases}
$$

where $k$ is the unit vector in the linc-of-centers of colliding molecules $A$ modelled as hard spheres with a diameter $d_{A}, s_{F}$ is the steric factor accounting for the fact that in order to have the reactive collisions it is necessary to lave a peculiar orientation of the colliding molecules, $g^{*}$ is the threshold relative velocity connected with the threshold energy of the reaction (1) by the relation

$$
E^{*}=m_{A} g^{* 2} / 4
$$

where $m_{A}$ is the molecular mass. We introduce the reduced dimensionless threshold energy $\varepsilon^{*}$

$$
\varepsilon^{*}=E^{*} / k_{\mathrm{B}} T \text {. }
$$

Introducing for $\sigma_{A A_{1}}^{*}$ the line-of-centers model (see Eq. (33)) we perform the calculations described at the end of Sec. 3. For the coefficients $a_{A}$ (see Eqs. (9), $(10),,(23)$ and $(24))$ we obtain

$$
a_{A}^{(1)}(1)=a_{A_{1}}^{(1)}(1)=\frac{1}{2} s_{F}\left(\varepsilon^{*}+\frac{1}{2}\right) \exp \left(-\varepsilon^{*}\right),
$$


where

$$
\begin{aligned}
& a_{A}^{(1)}(1,2)=a_{A_{1}}^{(1)}(1,2)=\frac{1}{24} s_{\mathrm{F}}\left(13 \varepsilon_{1}-\varepsilon_{2}\right) \exp \left(-\varepsilon^{*}\right), \\
& a_{A}^{(2)}(1,2)=a_{A_{1}}^{(2)}(1,2)=\frac{1}{24} s_{\mathrm{F}}\left(\varepsilon_{1}-\varepsilon_{2}\right) \exp \left(-\varepsilon^{*}\right),
\end{aligned}
$$

$$
\begin{aligned}
& \varepsilon_{1}=\varepsilon^{*}+\frac{1}{2}, \\
& \varepsilon_{2}=\varepsilon^{* 2}-\varepsilon^{*}-\frac{1}{4}
\end{aligned}
$$

and symbols $(1)$ and $(1,2)$ in $a_{A}^{(i)}$ have been introduced to indicate the Sonine polynomials used in Eq. (23). ized by

The nonequilibrium effect due to the chemical reaction (1) can be character-

$$
\eta=1-k / k_{A}^{(0)}
$$

Thus, for the line-of-centers model (sce Eq. (33)) using Eqs. (31), (34) and (35) we obtain

$$
k_{A}^{(0)}=4 s_{\mathrm{F}} d_{A}^{2}\left(\pi k_{\mathrm{B}} t / m_{A}\right)^{1 / 2} \exp \left(-\varepsilon^{*}\right)
$$

and from Eqs. (41), and (31)-(36) we get

$$
\eta(1)=\frac{1}{2} s_{\mathrm{F}}\left(\varepsilon^{*}+\frac{1}{2}\right)^{2} \exp \left(-\varepsilon^{*}\right),
$$

whereas from Eqs. (41), (31)-(35) and (37)-(40) we obtain

$$
\begin{aligned}
\eta(1,2)= & \left\{\frac{1}{2} s_{\mathrm{F}} \varepsilon_{1}\left[\varepsilon_{1}+\frac{1}{30}\left(\varepsilon_{1}-\varepsilon_{2}\right)\right]-\frac{1}{60} s_{\mathrm{F}} \varepsilon_{2}\left(\varepsilon_{1}-\varepsilon_{2}\right)\right\} \exp \left(-\varepsilon^{*}\right) \\
& -\frac{1}{16} s_{\mathrm{F}}\left[\varepsilon_{1}+\frac{1}{30}\left(\varepsilon_{1}-\varepsilon_{2}\right)\right]^{2} \varepsilon_{2}\left[\operatorname{cxp}\left(-\varepsilon^{*}\right)\right]^{2} .
\end{aligned}
$$

In Eqs. (43) and (44), similarly as in Eqs. (36)-(38), we have introduced $(i, j)$ to denote the Sonine polynomials used in derivation. Additionally we introduce the value of the rate constant calculated according to Eq. (42) in which we replace the temperature $T$ by the Shizgal-Karplus temperature $T_{A}^{\text {SK }}$. Thus, we obtain

$$
k\left(T_{A}^{\mathrm{SK}}\right)=4 s_{\mathrm{F}} d_{A}^{2}\left(\pi k_{\mathrm{B}} T_{A}^{\mathrm{SK}} / m_{A}\right)^{1 / 2} \exp \left(-\varepsilon^{*} T / T_{A}^{\mathrm{SK}}\right) .
$$

Introducing a definition

$$
\eta\left(T_{A}^{\mathrm{SK}}\right)=1-k\left(T_{A}^{\mathrm{SK}}\right) / k^{(0)}
$$

we obtain from Eqs. (45) and (42) the following result:

$$
\eta\left(T_{A}^{\mathrm{SK}}\right)=1-\left(T_{A}^{\mathrm{SK}} / T\right)^{1 / 2} \exp \left[\left(1-T / T_{A}^{\mathrm{SK}}\right) \varepsilon^{*}\right] .
$$

As follows from Eqs. (27) and (36) the Shizgal-Karplus temperature is expressed as

$$
T_{A}^{\mathrm{SK}}=\left[1-\frac{1}{2} s_{\mathrm{F}}\left(\varepsilon^{*}+\frac{1}{2}\right) \exp \left(-\varepsilon^{*}\right)\right] T .
$$

Equations (43), (44), (47) and (48) are the main results of our theoretical treatment. These results can be compared with the traditional theoretical results and with the Monte Carlo computer simulations. 
5. Calculation of nonequilibrium effects of the chemical reaction with the total neglecting of the products of this reaction

In this section after showing the fundamental differences between our treatment and traditional treatments we present the formulas for $\eta$ obtained in the traditional way, which are necessary for comparison with our treatment.

The derivations presented in Secs. 3 and 4 have been performed with a partial neglecting of products $B$ of the reaction (1). The Boltzmann equation for the reagent $A$ (Eq. (2)) has been simplified by the neglecting of the third term accounting for the elastic collisions between molecules $A$ and $B$ (see Eqs. (2) and (8)). But the product $B$ has been included in the definition of the total temperature $T$ of the system and in the equation of the energy balance (see Eq. (22)). In the more traditional approach (see for example Refs. [1] and [5]) products $B$ are totally disregarded. It means that it is also assumed that

$$
T=T_{A}=\text { const. }
$$

From Eqs. (8) and (49) after taking into account the conservation of energy it can be shown that (see Refs. [1] and [5])

$$
a_{A}^{(1)}=0 \text {. }
$$

It means that in the expansion (23) not only the first term (see Eq. (26)) but also the second term should be disregarded. Calculations of the nonequilibrium effects under the assumption expressed by Eqs. (49) and (50) lead to the following results. The expression derived by Present [5] with the use of the Sonine polynomial $S_{1 / 2}^{(2)}\left(\mathcal{C}_{A}^{2}\right)$ is

$$
\eta_{\mathrm{P}}(2)=\frac{1}{32} s_{\mathrm{F}}\left(\varepsilon^{* 4}-2 \varepsilon^{* 3}+\frac{1}{2} \varepsilon^{* 2}+\frac{1}{2} \varepsilon^{*}+\frac{1}{16}\right) \exp \left(-\varepsilon^{*}\right),
$$

whereas that obtained with the use of the Sonine polynomials $S_{1 / 2}^{(2)}\left(\mathcal{C}_{A}^{2}\right)$ and $S_{1 / 2}^{(3)}\left(\mathcal{C}_{A}^{2}\right)$ (see Refs. $\left.[6,7]\right)$ is

$$
\begin{aligned}
\eta_{\mathrm{P}}(2,3)= & \frac{1}{7680} s_{\mathrm{F}}\left(\frac{32}{9} \varepsilon^{* 6}-\frac{128}{3} \varepsilon^{* 5}+\frac{1184}{3} \varepsilon^{* 4}-\frac{1904}{3} \varepsilon^{* 3}\right. \\
& \left.+138 \varepsilon^{* 2}+140 \varepsilon^{*}+17\right) \exp \left(-\varepsilon^{*}\right) .
\end{aligned}
$$

Equation (52) is very convenient for comparison with our results because, as analyzed by Shizgal and Karplus [7], the effect of introduction of further Sonine polynomials is small.

\section{The Monte Carlo numcrical simulations}

In this section we describe the numerical simulations which we use to obtain the results for comparison with the results following from the analytical expressions derived in Sec. 4. We apply the modified Nanbu-Babovsky Monte Carlo simulations scheme, which we have already used and described for various cases of translational energy relaxation in the dilute gas [20-22] as well as for chemical and thermal effects in the bimolecular chemical reaction in the dilute gas [23]. Because 
we have already extensively discussed the modified Nanbu-Babovsky direct simulation scheme (see Refs. [20], [21] and [23]) we describe here only the parameters introduced for the system.

We have used the following system for numerical simulations. A volume $V=$ $1666.7 \mathrm{~nm}^{3}$ contains 500 spheres $A$ and 0 spheres $B$ having diameters $d_{A}=0.35 \mathrm{~nm}$ and masses $m_{A}=16 \mathrm{~g} /$ mole. This corresponds to the packing fraction 0.0067348 . The equilibrium temperature is $300 \mathrm{~K}$. In reactive collision spheres $A$ change to spheres $B$ having diameters $d_{B}=0.35 \mathrm{~nm}$ and masses $m_{B}=16 \mathrm{~g} / \mathrm{mole}$. As the chemical reaction proceeds the number of spheres $A$ changes from $N_{A}=500$ to $N_{A}=500-N_{B}$ and the number $N_{B}$ increases in time which is described by time increment characterized by time steps $\Delta t=0.6941 \times 10^{-19} \mathrm{~s}$. As in the early stages of chemical reaction the fastest spheres $A$ change to spheres $B$ the average translational energy of $A$ and the temperature of the component $A$ decrease. In order to obtain a better accuracy we have performed in each simulation a large number of runs $R(R>1000$ for all cases and for very slow reactions even $R=40000$ ) and calculated the average values of $N_{A}, N_{B}, T_{A}$ and $T_{B}$ as well as the numbers of elastic and reactive collisions as functions of time in time steps. The temperature $T_{B}$ is simply related to $T_{A}$ because from Eq. (6) it follows that $N_{A} T_{A}+N_{B} T_{B}=N T$. We have performed simulations for various dimensionless threshold energies $\varepsilon^{*}$ and steric factors $s_{F}$. Typical changes of $N_{B}$ and $T_{A}$ in time are represented in Fig. 1.

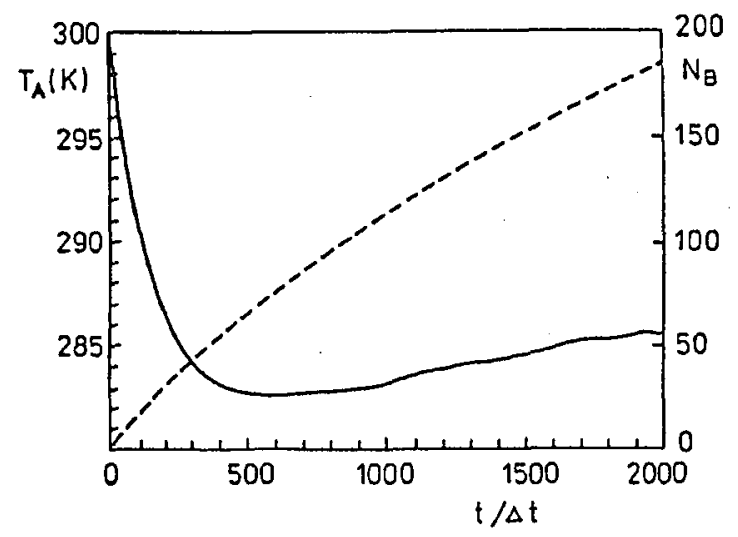

Fig. 1. The temperature $T_{A}$ (solid line) and number of particles $N_{B}$ of products (dashed line) as functions of time $t$ in time steps $\Delta t=0.6941 \times 10^{-19} \mathrm{~s}$ for the dimensionless threshold energy $\varepsilon^{*}=3$ (see Eqs. (33)-(35)) and the steric factor $s_{\mathrm{F}}=1$.

From Fig. 1 it can be seen that the so-called quasi-stationary state for the component $A$ characterized in a form of a long plateau for $T_{A}$ in the ranges of small $N_{B}$ can be obtained in the region of minimum of $T_{A}$. It should be emphasized that this region corresponds to small values of $N_{B}$ only for slow reactions, i.e. those characterized by sufficiently large $\varepsilon^{*}$ or small $s_{F}$. Therefore, comparison between the numerical results and those obtained from the perturbation theory should lead 
to reasonable results for slow chemical reactions only. We treat the minimum of $T_{A}$ as an approximation of the Shizgal-Karplus temperature $T_{A}^{\mathrm{SK}}$ (see Eqs. (27) and (48)) and we calculate the nonequilibrium value of the rate of chemical reaction (see Eqs. (29) and (30)) in the region of the above-mentioned plateau. The results of $\eta$ (see Eq. (41)) can be calculated from the values of the rate constant $k$ obtained from the simulations and those of $k^{(0)}$ obtained from Eq. (42).

\section{Comparison of the new and traditional analytical results with the results of numerical simulations}

In this section we compare the results for the relative change of the rate constant of chemical reaction represented by $\eta$ (see Eq. (41)). As $\eta$ is a linear function of the steric factor $s_{F}$, we present in Fig. $2 \eta / s_{F}$ as a function of the

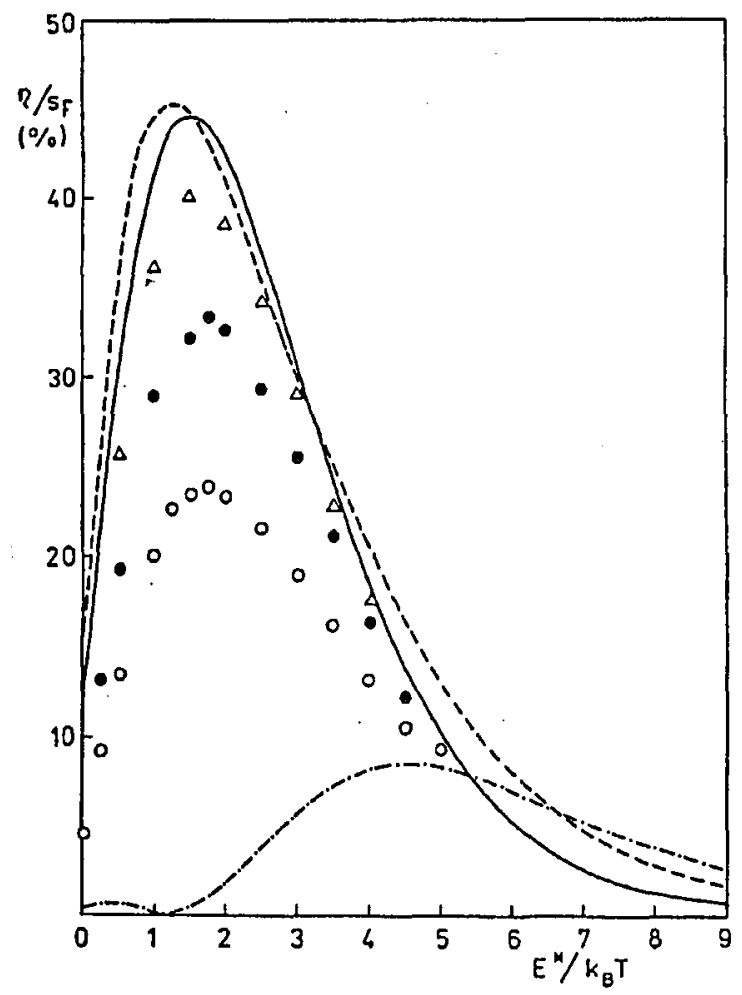

Fig. 2. Relative nonequilibrium corrections $\eta / s_{F}$ in percent (sce Eq. (41)) as a function of dimensionless threshold energy $\varepsilon^{*}$ (see Eqs. (33)-(35)). Analytical results calculated from $\eta(1)$ (see Eq. $(43)$ ), $\eta(1,2)$ (sce Eq. (44)) and $\eta_{\mathrm{p}}(2,3)$ (see Eq. (52)) are represented by solid, dashed, and dotted lines, respectively. Computer simulation results for the steric factors $s_{F}$ equal to $1,0.3333$, and 0.1 are represented by white circles, black circles and white triangles, respectively. 
dimensionless threshold energy $\varepsilon^{*}$ (see Eqs. (33)-(35)). We compare our analytical results (Eqs. (43) and (44)) with the traditional results (Eq. (52)) and with the results from our simulations performed for various steric factors. For the numerical results $\eta / s_{\mathrm{F}}$ is a function of $s_{\mathrm{F}}$. In order not to introduce too many results we do not present the curve obtained from Eq. (51) because this formula gives nearly the same results as Eq. (52). The results obtained from Eq. (47) do not represent a linear function of $s_{F}$ but they differ only slightly from those obtained from Eq. (43). As these small differences are the largest for $s_{F}=1$, in Fig. 3 we present $\eta\left(T_{A}^{\mathrm{SK}}\right)-\eta(1)$ as a function of $\varepsilon^{*}$ for $s_{F}=1$. It should be emphasized that for smaller values of $s_{F}$ these differences between the results obtained from Eqs. (47) and (43) are even smaller.

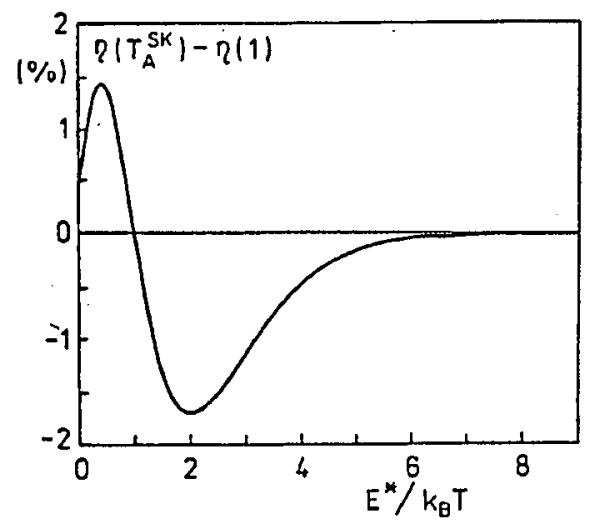

Fig. 3. The difference $\eta\left(T_{A}^{\mathrm{SK}}\right)-\eta(1)$ in percent (see Eqs. (47) and (43)) as a function of dimensionless threshold energy $\varepsilon^{*}$ (sce Eqs. (33)-(35)) calculated for $s_{F}=1$.

\section{Discussion and conclusions}

Results for the change of the rate constant of the chemical reaction (1) due to nonequilibrium effects have been analyzed. It is shown that, although in the perturbation solution of the Boltzmann equation for quasi-stationary state in which $n_{A} \gg n_{B}$ and the concentration of products $B$ can be neglected, even the small amount of products can have two important effects: (I) on the average kinetic energy of particles $A$ and (II) on the rate constant of chemical reaction. The effect (I) is connected with a temperature decrease resulting from the consumption of the fastest (the most energetic) particles $A$ in the chemical reaction. This effect represented by the Shizgal-Karplus temperature $T_{A}^{\mathrm{SK}}$ (see Eq. (48) and Fig. 1) has been already analyzed and compared with the results from the Monte Carlo simulations in our previous papers [23] and [27]. This effect cannot be compared with the results from the old treatments described in Sec. 5, because in such treatments $T_{A}$ is assumed to be constant and such an effect does not exist at all. The effect (II) represented by $\eta$ (see Eq. (41)) characterizing the relative change of 
the rate of chemical reaction due to nonequilibrium effects is completely different in our analytical results (see Eqs. (43), (41) and (47)) and in the results obtained from the traditional treatments (sce Eqs. (51), and (52)).

The new analytical results for $\eta$ (for $0<\varepsilon^{*}<4$ ) are not only much larger than the old ones but also the shapes of the curves representing $\eta$ as a function of $\varepsilon^{*}$ are completely different (see Fig. 2). It is also intercsting that the values for the first approximation of $\eta$ (sce Eq. (43)) and that directly calculated from the Shizgal-Karplus temperature (see Eqs. (47) and (48)) are nearly the same (see Fig. 3). The new values of $\eta / s_{F}$ are in reasonable agreement with the results obtained from the Monte Carlo simulations for sufficiently small values of the steric factor, i.e. for the reactions slow enough to be described by the perturbation method. That is why this agreement is better for $s_{F}=0.1$ than for $s_{F}=0.3333$ (see Fig. 2). We have also performed computer simulations for fast reactions, e.g. $s_{F}=1$ and $0<\varepsilon^{*}<2.5$. It is very interesting that even for these fast reactions for which the perturbation theory should not work we obtained smaller numerical results than the analytical ones but with the same shapes of curves representing $\eta / s_{\mathrm{F}}$ as a function of $\varepsilon^{*}$ as those following from our new results (Eqs. (43), (44) and (47)).

The results obtained in this paper liave been confirmed by additional investigations [28] and [29]. The results (obtained in Mimnesota University), based on the solution of the set of differential equations [28], permitted to get results even for $s \mathrm{~F}$ as small as 0.01 and 0.001 . The last results fully confirmed our perturbation solution of the Boltzmann equation. The authors of Ref. [28] were successful not only in the analysis of the role of neglecting of product concentration but also of the effect of neglecting of the reverse rcaction. In the meantime Górecki developed a phenomenological kinetic theory [29] in which this author showed that if $s_{\mathrm{F}} \rightarrow 0$ the result for the Shizgal-Karplus temperature (sce Eq. (48)) and for $\eta(1)$ (see Eq. (43)) should be exact. Naturally, the last treatment [29] is also a simplification because $\eta(1)$ for $0<\varepsilon^{*}<4$, even for the slowest reactions, gives good approximate results only. In this case a better approximation is obtained if more Sonine polynomials are considered, i.e. $\eta(1,2)(\mathrm{Eq} .(44))$ gives more exact results than $\eta(1)$ (Eq. (43)) and so on.

Just to summarize, in this paper we have prescnted analytical results from the new perturbation solution of the Boltzmann equation for the dilute gas with the chemical reaction $A+A \rightarrow B+B$ and we have verified them by the Monte Carlo computer simulations. The thicory has been more carcfully analyzed and the numerical simulations have been more accurately performed than in the introductory version presented in Świdno symposium in 1990 [23]. We have shown that in the isolated gaseous systems thie nonequilibrium effects preclicted by the perturbation solution of the Boltzmamn equation for the clicmical reaction $A+A \rightarrow B+B$ with inclusion of the energy transfer from reagents $A$ to products $B\left(n T=n_{A} T_{A}+n_{b} T_{B}\right)$ differ significantly from such effects calculated with the tolal neglecting of the product $B\left(T=T_{A}\right)$. We have obtained much larger effects for a relative cliange of the rate constant of the chemical reaction $\left(\eta / s_{F} \cong 45 \%\right.$ for $\left.\varepsilon^{*} \cong 1.5\right)$ than in the traditional treatments assuming $T_{A}=T$ and giving the maximum effect $\left(\eta / s_{F} \cong 8 \%\right.$ for $\varepsilon^{*} \cong 5$ ). These different maximum results are obtained in completely different 
ranges of $\varepsilon^{*}$, e.g. in old treatments for $\varepsilon^{*} \cong 1.5$ it was $\eta / s_{F}<1 \%$. We have also confirmed that introduction of the Shizgal-Karplus temperature is very convenient for the analysis of the nonequilibrium effects in such systems.

This paper cited as Ref. [13] in the recent paper [28] lias been prepared with a delay because of the unexpected death of Prof. J. Popielawski.

\section{Acknowledgments}

This research was supported in part by a grant from the Committee for Scientific Research.

\section{References}

[1] I. Prigogine, E. Xhrouet, Physica 15, 913 (1949).

[2] I. Prigogine, M. Malicu, Physica 16, 51 (1950).

[3] S. Chapman, T.G. Cowling, The Malhematical Theory of Nonuniform Gases, Cambridge University, Cambridge 1960.

[4] J.II. Ferziger, H.G. Kaper, Malhematical Theory of Transport Phenomena in Gases, North Holland, Amsterdam 1972.

[5] R.D. Present, J. Chem. Pliys. 31, 747 (1959).

[6] R.D. Present, J. Chem. Plyys. 48, 4875 (1968).

[7] B. Shizgal, M. Karplus, J. Chem. Phys. 52, 4262 (1970).

[8] J.M. Fitzpatrick, E.A. Desloge, J. Chem. Phys. 59, 5527 (1979).

[9] J. Popielawski, in: Dynamics of Systems wilh Chemical Reactions, Proc. Internat. Symp. Świdno (Poland), June 1988, Ed. J. Popielawski, World Sci. Publ., Singapore, New Jersey 1989, p. 95.

[10] A.S. Cukrowski, J. Popielawski, R. Sclmidt, W. Stiller, J. Chem. Phys. 89, 197 (1988).

[11] W. Stiller, R. Schmidt, J. Popielawski, A.S. Cukrowski, J. Chem. Phys. 93, 2425 (1990).

[12] A.S. Cukrowski, J. Popielawski, W. Stiller, R. Schmidt, J. Chem. Phys. 05, 6192 (1991).

[13] K. Nanbu, J. Phys. Soc. Jpn. 49, 2042 (1980).

[14] K. Nanbu, J. Phys. Soc. Jpn. 40, 2050 (1980).

[15] K. Nanbu, J. Phys. Soc. Jpn. 52, 3382 (1983).

[16] H. Babovsky, Math. Methods Appl. Sci. 8, 229 (1986).

[17] R. Illner, II. Neuzert, Transp. Theory Slat. Plyss. 16, 141 (1987).

[18] H. Ploss, Computing 38, 101 (1987).

[19] I.D. Boyd, J.P.V. Stark, J. Comput. Phys. 80, 374 (1989).

[20] S. Fritzsche, A.S. Cukrowski, Acta Phys. Pol. A 74, 811 (1988).

[21] A.S. Cukrowski, S. Fritzsche, Ann. Phys. (Leipzig) 48, 377 (1991).

[22] A.S. Cukrowski S. Fritzsche, J. Popielawski, Acta Phys. Pol. A 82, 1005 (1992).

[23] A.S. Cukrowski, S. Fritzsclie, J. Popielawski, in: Far-from-Equilibrium Dynamics of Chemical Systems, Proc. Second Inlernat. Symp. Świdno (Poland), Seplember 1990, Eds. J. Popielawski, J. Górecki, World Sci. Publ., Singapore, New Jersey 1991, p. 91. 
[24] J. Górecki, in: Far-from-Equilibrium Dynamics of Chemical Systems, Proc. Second Internat. Symp. Świdno (Poland), Septernber 1990, Eds. J. Popielawski, J. Górecki, World Sci. Publ., Singapore, New Jersey 1991, p. 133.

[25] J. Górecki, J. Popielawski, A.S. Cukrowski, Phys. Rev. A 44, 3791 (1991).

[26] J. Górecki, J. Chem. Plyss. 95, 2041 (1991).

[27] J. Popielawski, A.S. Cukrowski, S. Fritzsche, Physica A 188, 344 (1992).

[28] A.S. Cukrowski, J. Popielawski, Lilıong Qiı, J.S. Dahler, J. Chem. Phys. 07, 9086 (1992).

[29] J. Górecki, Pol. J. Chem. 6G, 1183 (1992).

[30] B. Shizgal, M. Karplus, J. Chem. Phys. 54, 4345 (1971). 